\title{
Transcriptomic analysis and identification of prognostic biomarkers in cholangiocarcinoma
}

\author{
HANYU LI ${ }^{1 *}$, JUNYU LONG $^{1 *}$, FUCUN XIE $^{1 *}, \mathrm{KAI} \mathrm{KANG}^{1}, \mathrm{YUE} \mathrm{SHI}^{1}$, WEIYU XU ${ }^{1}$, \\ XIAOQIAN WU ${ }^{1}$, JIANZHEN LIN ${ }^{1}$, HAIFENG XU ${ }^{1}$, SHUNDA DU ${ }^{1}$, YIYAO XU ${ }^{1}$, \\ HAITAO ZHAO $^{1}$, YONGCHANG ZHENG ${ }^{1}$ and JIN GU ${ }^{2}$ \\ ${ }^{1}$ Department of Liver Surgery, Peking Union Medical College Hospital, Chinese Academy of Medical Sciences \\ and Peking Union Medical College, Beijing 100730; ${ }^{2}$ MOE Key Laboratory of Bioinformatics, \\ BNIRST Bioinformatics Division, Department of Automation, Tsinghua University, Beijing 100084, P.R. China
}

Received February 8, 2019; Accepted August 20, 2019

DOI: $10.3892 /$ or.2019.7318

\begin{abstract}
Cholangiocarcinoma (CCA) is acknowledged as the second most commonly diagnosed primary liver tumor and is associated with a poor patient prognosis. The present study aimed to explore the biological functions, signaling pathways and potential prognostic biomarkers involved in CCA through transcriptomic analysis. Based on the transcriptomic dataset of CCA from The Cancer Genome Atlas (TCGA), differentially expressed protein-coding genes (DEGs) were identified. Biological function enrichment analysis, including Gene Ontology (GO) analysis and Kyoto Encyclopedia of Genes and Genomes (KEGG) pathway enrichment analysis, was applied. Through protein-protein interaction (PPI) network analysis, hub genes were identified and further verified using open-access datasets and qRT-PCR. Finally, a survival analysis was conducted. A total of 1,463 DEGs were distinguished, including 267 upregulated genes and
\end{abstract}

Correspondence to: Dr Yongchang Zheng, Department of Liver Surgery, Peking Union Medical College Hospital, Chinese Academy of Medical Sciences and Peking Union Medical College, 1 Shuaifu Road, Beijing 100730, P.R. China

E-mail: zhengyongchang@pumch.cn

Dr Jin Gu, MOE Key Laboratory of Bioinformatics, BNIRST Bioinformatics Division, Department of Automation, Tsinghua University, 30 Shuangqing Road, Beijing 100084, P.R. China

E-mail: jgu@tsinghua.edu.cn

*Contributed equally

Abbreviations: CCA, cholangiocarcinoma; TCGA, The Cancer Genome Atlas; DEGs, differentially expressed protein-coding genes; GO, Gene Ontology; KEGG, Kyoto Encyclopedia of Genes and Genomes; PPI, protein-protein interaction; GEO, Gene Expression Omnibus

Key words: transcriptomic analysis, hub genes, differentially expressed protein-coding genes, cholangiocarcinoma, prognosis, biomarker
1,196 downregulated genes. For the GO analysis, the upregulated DEGs were enriched in 'cadherin binding in cell-cell adhesion', 'extracellular matrix (ECM) organization' and 'cell-cell adherens junctions'. Correspondingly, the downregulated DEGs were enriched in the 'oxidation-reduction process', 'extracellular exosomes' and 'blood microparticles'. In regards to the KEGG pathway analysis, the upregulated DEGs were enriched in 'ECM-receptor interactions', 'focal adhesions' and 'small cell lung cancer'. The downregulated DEGs were enriched in 'metabolic pathways', 'complement and coagulation cascades' and 'biosynthesis of antibiotics'. The PPI network suggested that $C D K 1$ and another 20 genes were hub genes. Furthermore, survival analysis suggested that CDK1, MKI67, TOP2A and PRCl were significantly associated with patient prognosis. These results enhance the current understanding of CCA development and provide new insight into distinguishing candidate biomarkers for predicting the prognosis of CCA.

\section{Introduction}

Cholangiocarcinoma (CCA) originates from intrahepatic or extrahepatic bile duct epithelial cells and is classified into intrahepatic CCA (iCCA), perihilar CCA (pCCA), and distal CCA (dCCA) according to the anatomic location $(1,2)$. Among the majority of CCA tumors, pCCA accounts for $60-70 \%$, dCCA accounts for $20-30 \%$ and iCCA accounts for $5-10 \%$. CCA is recognized as the second most commonly diagnosed primary liver tumor and accounts for approximately $1-15 \%$ of all hepatobiliary malignancies (3). Although the average incidence of CCA is low, early diagnosis and treatment of CCA are difficult, and the overall patient prognosis is poor (4). Recently, iCCA has become the leading cause of death related to primary liver tumor (5). Systemic drug therapy is currently limited for patients with advanced or metastatic CCA, while surgical treatment is suitable only for those with early-stage CCA, which has a high risk of recurrence $(6,7)$. The median survival time of patients with advanced CCA is less than 2 years, and the 5-year survival rate is only $10 \%(3,8)$. Searching for genetic drivers that affect the occurrence and progression of CCA is important for exploring the molecular diagnosis and 
targeted therapy (1). In recent years, biomarker research has achieved progress in the prediction, treatment and prognosis of CCA. For example, KRAS mutations and PRKACB fusion genes have been identified in pCCA and dCCA, and somatic mutations of isocitrate dehydrogenase (IDH) have been identified in iCCA (9). In addition, inducible nitric oxide synthase (iNOS) has been involved in the occurrence of CCA through an inflammation-dependent manner (10). However, due to strong genetic heterogeneity, the current understanding of the molecular mechanisms of CCA is still not comprehensive. In particular, understanding of the genetic variations that promote CCA initiation and development are still fragmented. Moreover, the key driver genes of carcinogenesis remain unknown $(4,11)$. Therefore, studying the pathogenesis of CCA and identifying hub genes that are involved in the development of CCA remain a major challenge.

The Cancer Genome Atlas (TCGA) is a publicly sponsored project with the purpose of classifying and identifying major carcinogenic genomic alterations among large cohorts of more than 30 human tumors. To perform an integrated analysis of cancer genome profiles, high-throughput technologies relying on the use of microarrays and next-generation sequencing methods were applied in TCGA (12). RNA sequencing (RNAseq) has become useful for transcriptome (total RNA) profiling and obtaining accurate strand information. RNAseq is a method that is conductive to the application of a systematic comprehensive study of differentially expressed gene interactions and related signaling pathways with high precision. Moreover, protein-protein interaction (PPI) networks are useful for distinguishing hub genes, which are defined as genes with a high degree of connectivity that play an essential role in stabilizing the PPI network structure $(13,14)$. There are numerous oncology studies based on TCGA. From the perspective of CCA, Wang et al thoroughly studied the lncRNA-miRNA-mRNA ceRNA network and identified three IncRNAs, COL18A1-AS1, SLC6A1-AS1 and HULC, as being significantly associated with overall CCA patient survival (15). However, in the present study, we focused on identifying hub genes within the PPI and exploring their potential roles in CCA on the basis of TCGA combined with multiple datasets.

In the present research, transcriptomic iCCA data from TCGA were utilized to identify differentially expressed protein-coding genes (DEGs) between iCCA and normal tissues. Then, we executed Gene Ontology (GO) and Kyoto Encyclopedia of Genes and Genomes (KEGG) enrichment analysis to study alterations in biological functions and signaling pathways of iCCA. PPI network construction was performed, followed by identification of hub genes. Moreover, we identified the differential expression of hub genes by analyzing transcriptomic CCA data from several open-access databases, including Gene Expression Omnibus (GEO) database and ArrayExpress Archive of Functional Genomics Data (ArrayExpress). Further, we performed quantitative polymerase chain reaction (qPCR) in the laboratory to verify these hub genes. Finally, we executed survival analysis of the identified hub genes. The objective of this study was to understand CCA carcinogenesis by exploring the genetic changes involved in disease progression and to identify potential biomarkers that may be helpful for predicting the prognosis of iCCA patients.

\section{Materials and methods}

Acquisition of transcriptome data and identification of DEGs. Data for CCA mRNA expression were downloaded from TCGA database (https://portal.gdc.cancer.gov/, RNA-seq, Illumina) on July 6, 2018. Practical Extraction and Reporting Language (Perl) was utilized for sample information extraction, mRNA expression matrix generation, and gene symbol annotation. Only samples of primary site of liver and intrahepatic bile ducts were included for subsequent analysis, Statistical softwareR (version 3.4.4) and the 'DEseq' package from Bioconductor were used to perform significance analysis of the DEGs between CCA samples and adjacent noncancerous tissues $(16,17)$. Genes with an absolute value of $\log 2$ fold change $(\log 2 \mathrm{FC})>2$ and a corrected $\mathrm{P}$-value $<0.0001$ were defined as DEGs.

Functional and pathway enrichment analyses. GO term enrichment analysis was applied to analyze the biological significance of DEGs, which includes biological processes (BP), cellular components (CC) and molecular functions (MF), based on the GO online platform David (https://david.ncifcrf.gov/, date of access: 2019/5/7, species: Human). GO visualization was achieved by the R package 'GOplot' (18). KEGG pathway enrichment analysis was applied based on the online platform David. Critical pathways enriched in DEGs were identified. Visualization of KEGG results was conducted by $\mathrm{R}$ package 'ggplot2'. $\mathrm{P}<0.05$ was considered statistically significant for both GO and KEGG analysis.

PPI network analysis. Proteins usually perform biological functions synergistically. Strong relationships have been shown to exist between PPIs and the biological functions of gene/protein clusters (19). Therefore, PPIs must be explored by considering functional groups. PPI network analysis is helpful for distinguishing hub genes among a cluster of DEGs that are implicated in CCA progression based on their interaction levels. PPI information of DEGs was obtained from the STRING database; highest confidence of 0.900 was chosen (version 11.0, https://string-db.org/, data of access: 2019/5/7). The PPI networks for upregulated genes was constructed using Cytoscape3.6.1 software (https://cytoscape.org/). The top 15 genes with the highest degree of connectivity were defined as hub genes.

Identification of hub genes. CCA-related transcriptomic datasets were obtained from GEO (GSE76297 and GSE26566) and ArrayExpress (E-GEOD-32879 and E-GEOD-45001) (20-24). $\mathrm{R}$ (version 3.4.4) and the 'Limma' package of Bioconductor were used for identification of the DEGs (25). A P-value $<0.05$ was considered statistically significant. Finally, hub genes in TCGA were compared with the DEGs acquired from other 4 datasets. Hub genes with similar differential expression among 5 datasets were selected for further analysis. A Venn diagram indicating the intersection of multiple datasets was made online (http://bioinformatics.psb.ugent. be/cgi-bin/liste/Venn/calculate_venn.htpl).

Statistical analysis. To examine the classification effect of hub genes on cholangiocarcinoma and normal tissue, receiver 
operating characteristic (ROC) curves and area under the curve (AUC) were calculated by R package 'pROC' (26). Furthermore, clinical data of iCCA were downloaded from TCGA. We divided patients into two groups based on tumor stage: Stage I+II and stage III+IV. The association between hub gene expression and tumor stage was evaluated by Mann-Whitney $\mathrm{U}$ test. A P $<0.05$ was considered statistically significant. Finally, 'survival' package of Bioconductor was used to generate overall survival curves $(27,28)$. Perl was used to extract the lifetime of each patient from the clinical cart downloaded from TCGA. Clinical data of 118 patients with cholangiocarcinoma were downloaded from PubMed Central (11). Matching sample information was obtained from GEO dataset: GSE89749 (11). For each dataset, the patients were divided into two groups using the median gene expression value as the cut-off value. The relationship between patient overall survival and expression level of hub genes was tested by Cox proportional-hazards model. $\mathrm{P}<0.05$ was considered statistically significant.

$R T$ - $q P C R$. Tissue samples were collected as pairs, i.e., tumor tissue and adjacent normal tissue, from 10 patients with iCCA undergoing surgery at Peking Union Medical College Hospital. A total of 6 male and 4 female patients with mean age of 62 (range, 54-68) years were included. The collected tissue samples were stored in a refrigerator at $-80^{\circ} \mathrm{C}$. All patients were enrolled from November 2018 to April 2019. The study was approved by the Clinical Research Ethics Committee of Peking Union Medical College Hospital. Each patient provided a written informed signed consent. Total RNA was isolated from each sample with Trizol LS reagent (Invitrogen; Thermo Fisher Scientific, Inc.), and then used for cDNA synthesis using oligo(dT)primers and SuperScript $^{\mathrm{TM}}$ III Reverse Transcriptase (Invitrogen; Thermo Fisher Scientific, Inc.). PCR Master Mix (2X) (Superarray) and Applied Biosystems QuantStudio5 Real-time PCR system (Thermo Fisher Scientific, Inc.) were utilized for RT-qPCR. The sequences of primers for selected hub genes and housekeeping gene ( $\beta$-actin) are shown in Table SI.

Visualization of differential expression. For hub genes validated by qRT-PCR, R package 'ggpubr' (https://rpkgs. datanovia.com/ggpubr/index.html) was used to visualize gene expression based on the expression profile of DEGs in TCGA and the results of qRT-PCR. A t-test was used to calculate differences between groups. $\mathrm{P}<0.05$ was considered statistically significant.

\section{Results}

Identification of DEGs in CCA and normal tissues. The transcriptomic dataset of CCA and the corresponding clinical cart were downloaded from the TCGA database. Patients with lesion of primary site of liver and intrahepatic bile ducts, i.e., patients with iCCA, were included for further analysis. Therefore, a total of 33 cases were acquired, including 19 female and 14 male patients. Forty-one samples were acquired in total, including 33 tumor tissue samples and 8 normal tissue samples. A total of 1,463 DEGs $(\log 2 \mathrm{FC}>2$, corrected $\mathrm{P}<0.0001)$ were acquired, including 267 significantly upregulated DEGs and 1,196 significantly downregulated DEGs. A heatmap and a volcano plot showing the expression levels of these genes are shown in Fig. 1.

Functional and pathway enrichment analyses of DEGs. GO and KEGG pathway enrichment analyses were conducted to explore the functional characteristics of the DEGs. The GO analysis results revealed that the upregulated DEGs were significantly enriched in 'extracellular matrix organization', 'cell-cell adhesion', 'cell adhesion', 'epithelial cell morphogenesis involved in placental branching and mitotic spindle assembly' in terms of BP. Regarding MF, the upregulated DEGs were enriched in 'cadherin binding involved in cell-cell adhesion', 'structural molecule activity', 'collagen binding', 'protein binding' and 'signal transducer activity'. Under CC, the upregulated DEGs were enriched in 'cell-cell adherens junction', 'extracellular exosome', 'midbody, cell-cell junction' and 'cytoplasmic microtubule'. For the downregulated DEGs, significant enrichment was observed in the 'oxidation-reduction process', 'xenobiotic metabolic process', 'metabolic process', 'steroid metabolic process' and 'platelet degranulation' under BP. For MF, the downregulated DEGs were significantly enriched in 'oxidoreductase activity', 'monooxygenase activity', 'iron ion binding', 'oxidoreductase activity acting on paired donors, with the incorporation of or reduction in molecular oxygen' and 'electron carrier activity'. For CC, the DEGs were enriched in 'extracellular exosome', 'blood microparticle', 'organelle membrane', 'mitochondrial matrix' and 'extracellular region'. In addition, the KEGG analysis results showed that the upregulated DEGs were significantly enriched in 'ECM-receptor interactions', 'focal adhesion', 'small cell lung cancer', 'pathways in cancer' and 'hypertrophic cardiomyopathy (HCM)'. Meanwhile, the downregulated DEGs were enriched in 'metabolic pathways', 'complement and coagulation cascades', 'biosynthesis of antibiotics', 'retinol metabolism' and 'fatty acid degradation'. The enriched GO terms and KEGG pathways are shown in Fig. 2 and Tables SII-SV.

Construction of the PPI network and hub gene identification. PPI network analysis can be used to distinguish critical hub genes among a group of DEGs. Therefore, the STRING database was used to conduct the PPI network analysis. PPI networks for the upregulated genes were constructed by Cytoscape 3.6.1 (Fig. 3).

Cytoscape 3.6.1 was used to perform a centrality analysis. The top 15 genes with the highest degree of connectivity were defined as hub genes. Under this criterion, 15 hub genes were obtained for the upregulated DEGs, including cyclin-dependent kinase $1(C D K 1)$, cyclin B2 (CCNB2), kinesin family member $2 \mathrm{C}(K I F 2 C)$, topoisomerase (DNA) II $\alpha(T O P 2 A)$, centrosomal protein 55 (CEP55), ribonucleotide reductase regulatory subunit M2 (RRM2), ubiquitin conjugating enzyme E2 C (UBE2C), baculoviral IAP repeat containing 5 (BIRC5), centromere protein $\mathrm{F}(C E N P F)$, NIMA-related kinase 2 (NEK2), forkhead box M1 (FOXM1), marker of proliferation Ki-67 (MKI67), protein regulator of cytokinesis 1 (PRC1), integrin subunit $\alpha 2$ (ITGA2), and laminin subunit $\gamma 2$ (LAMC2). Hub genes for the downregulated DEGs consisted of kininogen 1 (KNGl), complement $\mathrm{C} 3(C 3)$, apolipoprotein A1 (APOAl), albumin 

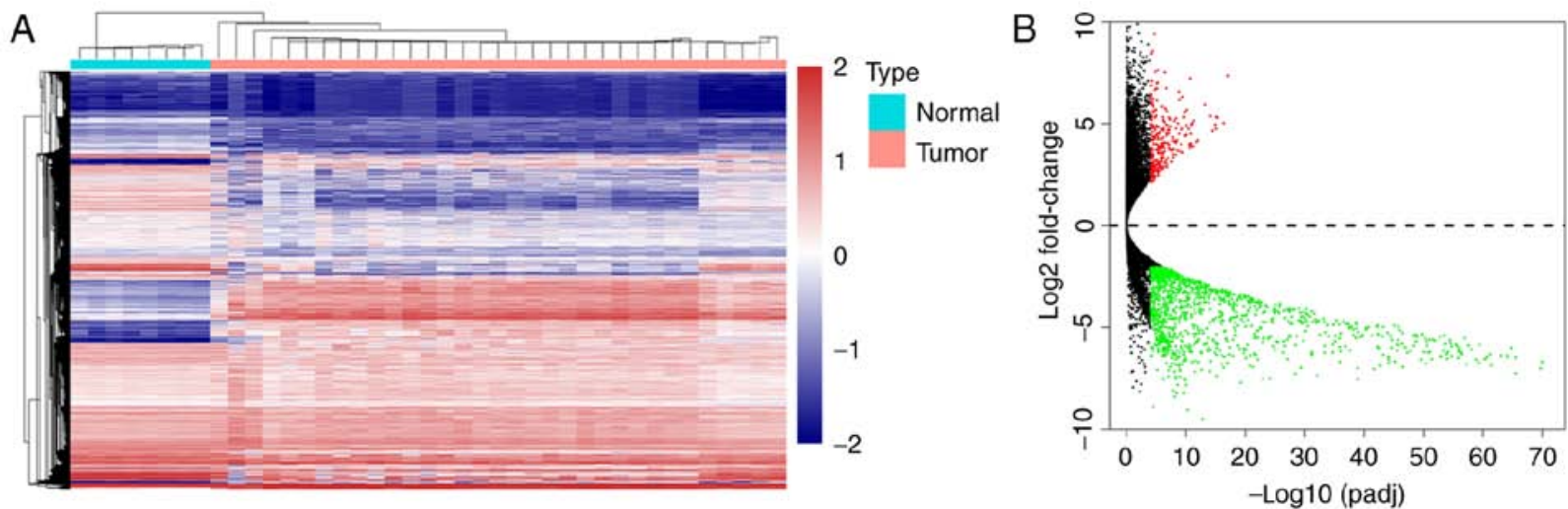

Figure 1. Heatmap and volcano plot showing significant DEGs between 33 iCCA tissues and 8 normal tissues in TCGA. (A) Rows represent genes, and columns represent samples. (B) The red spots represent significantly upregulated genes, and the green spots represent significantly downregulated genes. TCGA, The Cancer Genome Atlas; DEGs, differentially expressed protein-coding genes; CCA, cholangiocarcinoma; iCCA, intrahepatic CCA.
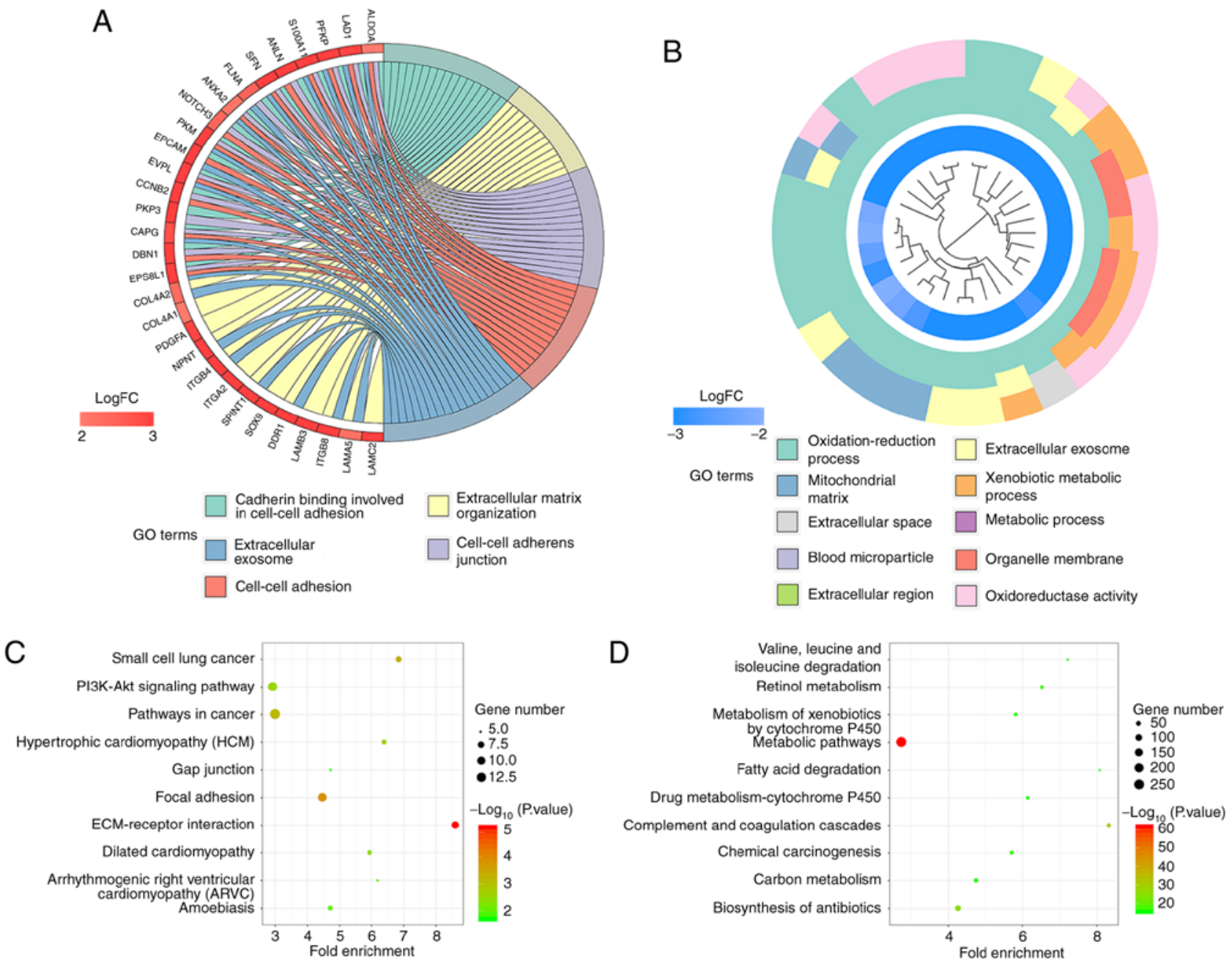

Figure 2. Functional enrichment analysis of DEGs. (A) GO cluster plot showing a chord dendrogram of the clustering of the expression spectrum of significantly upregulated genes. (B) GO cluster plot showing a circular dendrogram of the clustering of the expression spectrum of significantly downregulated genes. KEGG pathway enrichment dot plot of the (C) significantly upregulated genes and (D) downregulated genes. The y-axis represents KEGG-enriched terms. The $\mathrm{x}$-axis represents the fold of enrichment. The size of the dot represents the number of genes under a specific term. The color of the dots represents the adjusted P-value. DEGs, differentially expressed protein-coding genes; GO, Gene Ontology; KEGG, Kyoto Encyclopedia of Genes and Genomes.

$(A L B)$, fibrinogen $\alpha$ chain $(F G A)$, apolipoprotein $\mathrm{B}(A P O B)$, fibrinogen $\gamma$ chain $(F G G)$, 3-hydroxyacyl CoA dehydrogenase $(E H H A D H), \alpha 2-\mathrm{HS}$ glycoprotein $(A H S G)$, apolipoprotein A2
(APOA2), complement $\mathrm{C} 4 \mathrm{~A}(C 4 A)$, serpin family A member 1 (SERPINA1), apolipoprotein E (APOE), serpin family C member 1 (SERPINCI) and acyl-CoA oxidase 1 (ACOXI). 

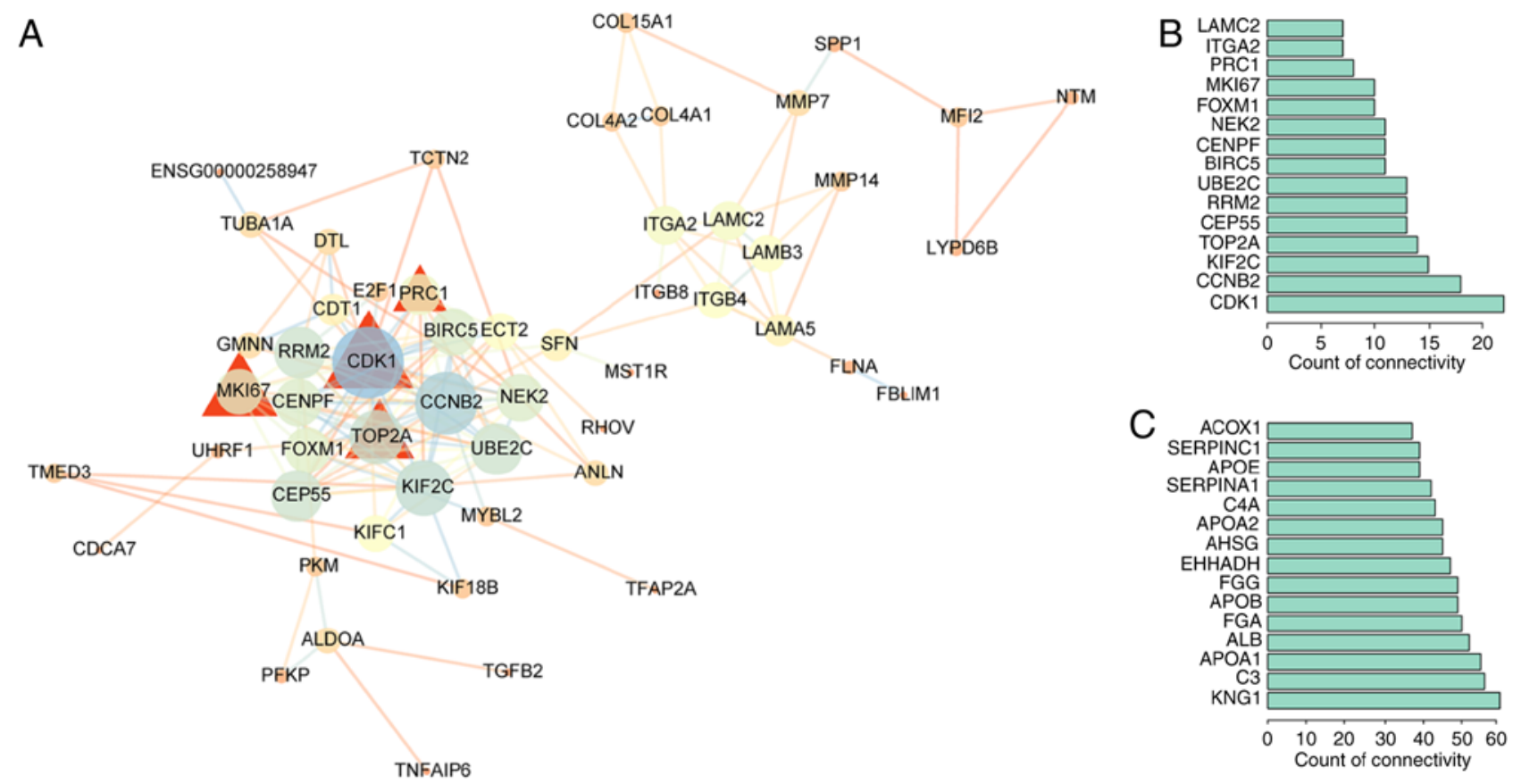

Figure 3. (A) PPI network of the significantly upregulated DEGs. The nodes represent the significantly upregulated DEGs. The edges represent the interaction of significantly upregulated DEGs. The triangles represent hub genes validated by qRT-PCR. Bar chart of the (B) upregulated hub genes and the (C) downregulated hub genes. The x-axis represents count of connectivity. The y-axis represents hub gene symbols. PPI, protein-protein interaction; DEGs, differentially expressed protein-coding genes.

To further verify the differential expression of the critical hub genes in CCA, we evaluated the expression profiles of 30 hub genes in another 4 datasets. GSE76297 consists of 304 specimens in total, 183 of which were utilized in analysis, including $91 \mathrm{CCA}$ tumor tissues and $92 \mathrm{CCA}$ non-tumor tissues. GSE26566 consists of 169 specimens in total, 163 of which were utilized in analysis, including 104 CCA tissues and 59 surrounding liver tissues. E-GEOD-32879 consists of 37 specimens in total, 23 of which were utilized in analysis, including 16 iCCA tissues and 7 non-tumor tissues. E-GEOD-45001 consists of 10 pairs of iCCA tumor tissues and non-tumor tissues, which were all utilized in analysis. Consistent with our results, 21 out of 30 hub genes in TCGA were found to share similar differential expression among the other 4 datasets, including 8 upregulated hub genes and 13 downregulated hub genes (Fig. S1). The differential expression of hub genes is shown in Fig. 4A-D.

ROC curves and tumor staging correlation analysis. ROC curves for hub genes were generated based on expression profile of TCGA dataset. AUC was $>0.900$ for all 21 selected hub genes (Fig. S2). Among them, the expression of 5 downregulated hub genes, including $A C O X 1, A P O A 2, A P O B, F G A$ and $F G G$, was inversely associated with tumor stage $(\mathrm{P}<0.05$, Fig. S3). For other identified hub genes, no association between gene expression and tumor stage was found.

Survival analysis. For upregulated hub genes, the expression of $C D K 1, M K I 67, T O P 2 A$ and $P R C 1$ was negatively related to the overall survival time of CCA patients in both TCGA and GSE89749 datasets $(\mathrm{P}<0.05)$. No significant result was found for the downregulated hub genes. The survival curves are shown in Fig. 5.
Identification of CDK1, MKI67, TOP2A and PRC1 by $R T-q P C R$. Since the survival analysis indicates that the overexpression of $C D K 1, M K I 67, T O P 2 A$ and $P R C 1$ predicts poor survival of patients with cholangiocarcinoma, we performed RT-qPCR to validate the expression change of these genes in frozen tissue. As expected, all of the 4 genes were upregulated in the iCCA tissue (Fig. 4E-H).

\section{Discussion}

Cholangiocarcinoma (CCA) is recognized as the second most commonly diagnosed primary liver tumor. Due to its strong genetic heterogeneity, the current understanding of the pathogenesis of CCA is not comprehensive. Concerning genetic changes involved in CCA initiation and progression, agreement in this field remains fragmented. The key drivers involved in CCA carcinogenesis still need to be defined $(3,4,11)$. In the present study, we focused on the genetic changes in transcription level between intrahepatic CCA (iCCA) and normal tissue. A total of 1,463 differentially expressed protein-coding genes (DEGs) were obtained based on data from The Cancer Genome Atlas (TCGA). Gene Ontology (GO) enrichment analyses showed that 'changes in cadherin binding involved in 'cell-cell adhesion', 'extracellular matrix organization' and the 'cell-cell adherens junction' represented significant GO terms for the upregulated DEGs and that 'oxidation-reduction processes', 'extracellular exosomes', and 'blood microparticles' represented significant GO terms for the downregulated DEGs. In addition, 'ECM-receptor interactions', 'focal adhesions' and 'small cell lung cancer' were significant pathways related to the upregulated DEGs. 'Metabolic pathways', 'complement and coagulation cascades' and 'biosynthesis of antibiotics' were significant pathways for the downregulated DEGs. 

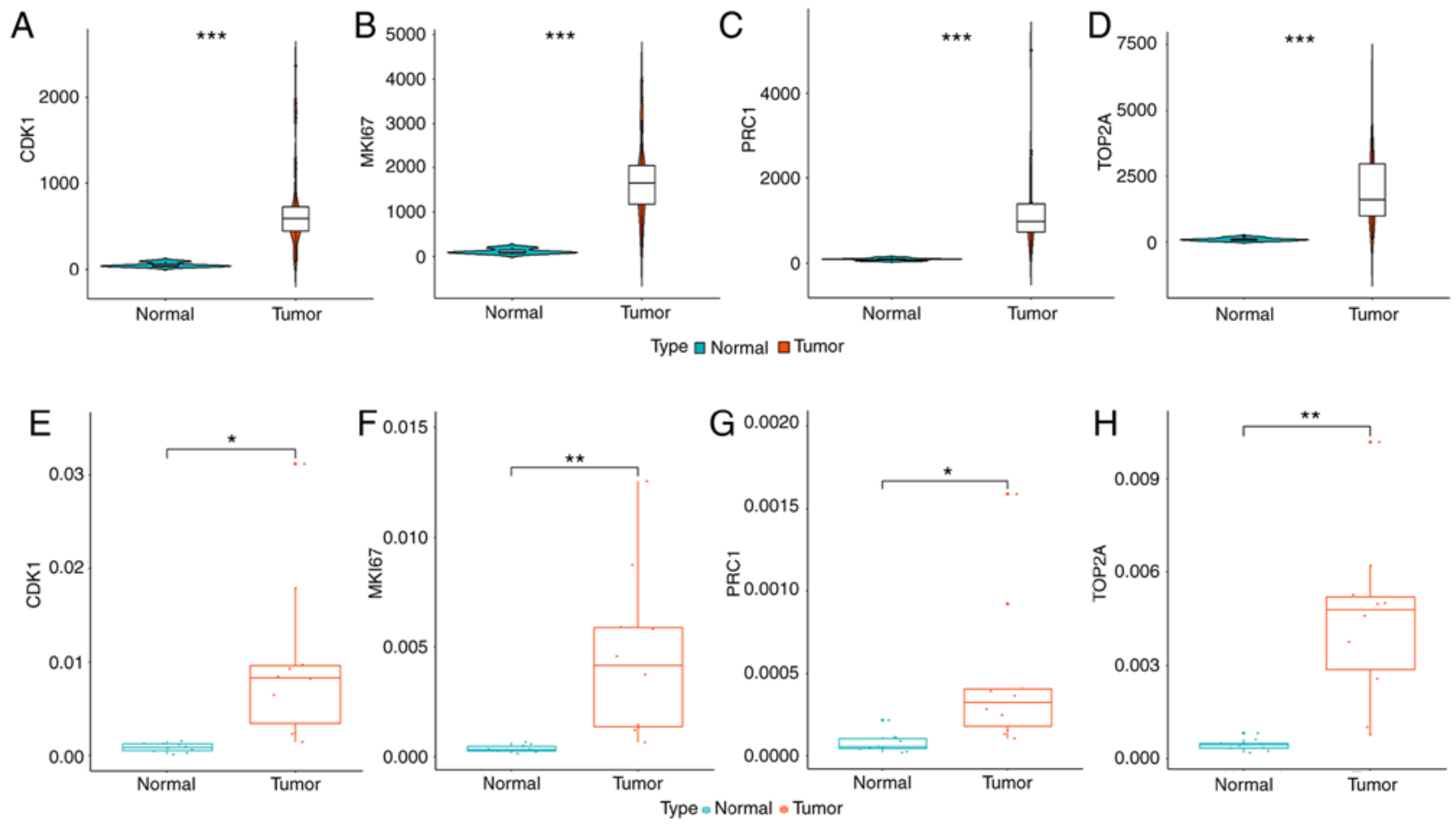

Figure 4. Dynamic expression of significantly upregulated hub genes. (A-D) Expression of hub genes from the TCGA database. (E-H) Relative quantification of hub genes based on qRT-PCR results. ${ }^{*} \mathrm{P}<0.05,{ }^{* * *} \mathrm{P}<0.01,{ }^{* *} \mathrm{P}<0.001 . C D K 1$ (A and E), MKI67 (B and F), PRC1 (C and G) and TOP2A (D and H). TCGA, The Cancer Genome Atlas; CDK1, cyclin dependent kinase 1; MK167, marker of proliferation Ki-67; PRC1, protein regulator of cytokinesis 1; TOP2A, DNA topoisomerase II $\alpha$.

Hub genes were identified based on the degree of connectivity. Fifteen upregulated hub genes and 15 downregulated hub genes were selected based on protein-protein interaction (PPI) network. Moreover, the expression profiles of the 30 hub genes were verified using datasets from GEO and Arrayexpress. A total of 21 hub genes showed stable differential expression among 5 datasets including TCGA. ROC curves revealed that all 21 hub genes presented a credible classification effect between tumor and normal tissue with AUC $>0.900$. In addition, the expression of $A C O X 1, A P O A 2, A P O B, F G A$ and $F G G$ was inversely associated with tumor stage, which indicates that these genes may be involved in the progression of CCA.

To further explore the relationships between hub genes and the outcomes of CCA patients, a survival analysis was conducted based on the clinical data and expression profiles of the identified hub genes in both TCGA and GSE89749. Four upregulated hub genes, including $C D K 1, M K I 67, T O P 2 A$ and $P R C l$, were identified as being significantly related to overall survival among CCA patients. Moreover, the differential expression of the four genes was validated by RT-qPCR. Therefore, we considered CDK1, MKI67, TOP2A and PRC1 as potential predictors for the poor prognosis of patients with CCA.

Cyclin-dependent kinases (CDKs) are a family of protein kinases driving the major events of cell cycle control (29). Aberrant expression of CDK1 is involved in cell cycle arrest in many tumor types such as melanoma, colon cancer and pancreatic cancer (30). Studies that have focused on the roles of CDK1 in cholangiocarcinoma are limited. Okumura et al revealed that CDK1 is upregulated by AIB1, i.e., transcriptional coactivator amplified in breast cancer 1 , through the Akt pathway. AIB1 was found to be overexpressed in human CCA specimens and promote cell cycle progression at the $\mathrm{G} 2 / \mathrm{M}$ phase by inducing CDK1 (31). In addition, CDK1 may be involved in drug-resistant mechanisms of CCA since western blot analyses indicated that $\mathrm{G} 2 / \mathrm{M}$ phase-regulated proteins, including CDK1, were downregulated in gemcitabine-resistant CCA cell lines (32).

MK167 encodes Ki-67. It is a cell cycle-regulated phosphatase 1-binding protein universally used as a proliferation marker. $\mathrm{Ki}-67$ is a major organizer required for assembly of the perichromosomal compartment in cells (33). The Ki-67 index is shown to be the most reliable prognostic evaluation factor of gastroenteropancreatic neuroendocrine neoplasms (GEP-NENs) (34). The Ki-67 index can be variable through the disease course (35-37). In combination with tumor type, site and stage, the Ki-67 index is used to stratify patients in different prognostic categories (34). In the present study, we found a prognostic role of MKI67 in CCA. This finding may be clinically valuable, although the underlying mechanisms for Ki-67 variation still requires further investigation.

DNA topoisomerase II $\alpha$ (TOP2A) is an isoform of DNA topoisomerase II (Topo II). Topo II is a crucial enzyme for cell division that generates torsional stress on double-stranded DNA by inducing transient breaks that are subsequently resealed (38). TOP2A is located adjacent to the HER2 oncogene and is frequently coamplified with HER 2 in multiple types of cancers, such as breast cancer, bladder cancer and gastric adenocarcinoma (39-42) However, Panvichian et al reported that TOP2A overexpression in hepatocellular carcinoma (HCC) is independent of HER2 gene amplification or expression (43). Our results showed that TOP2A is significantly 

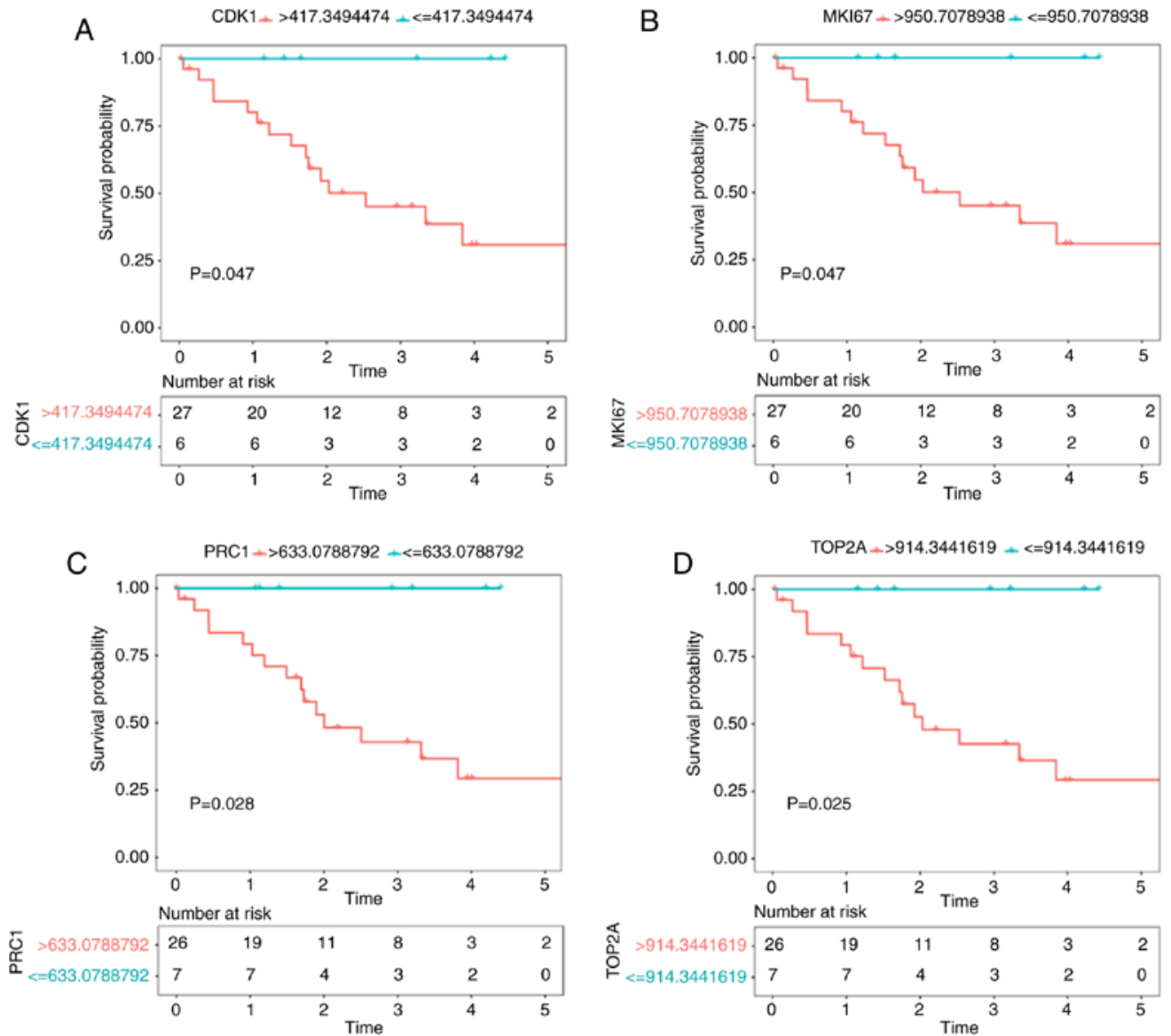

$E$
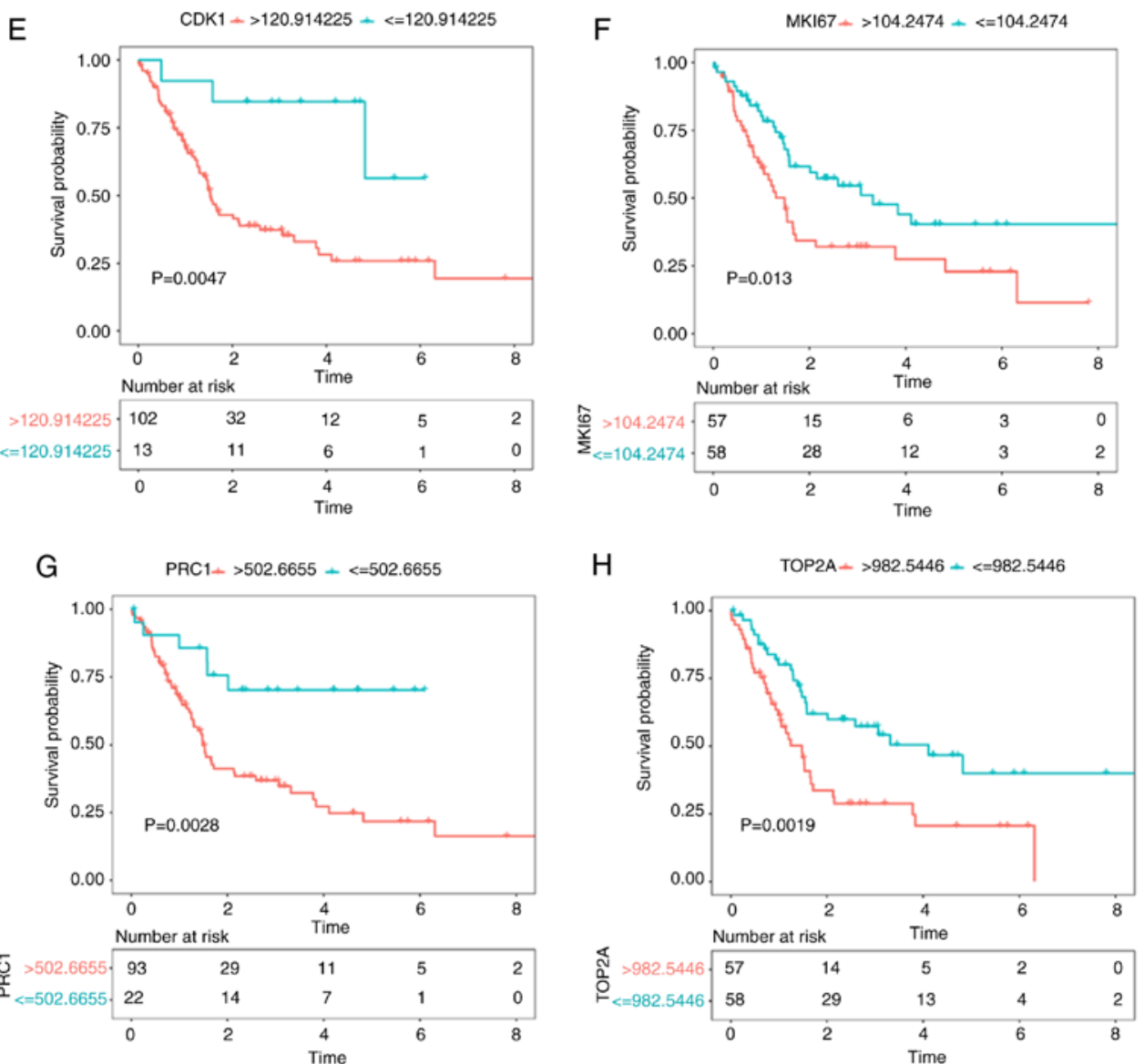

Figure 5. Survival analysis of significantly upregulated hub genes. CDK1 (A and E), MKI67 (B and F), PRC1 (C and G), TOP2A (D and H). A-D refer to survival curves based on TCGA. E-H refer to survival curves based on GSE89749. Overall survival time is recorded in years. The cut-off value is the median gene expression. TCGA, The Cancer Genome Atlas. 
upregulated in CCA tissues and represents a possible predictive biomarker for poor prognosis. However, no significant change was detected in the transcription of HER2. Therefore, TOP2A may play a role in CCA tumorigenesis independent of HER2. Nateewattana et al reported that andrographolide, a Topo II inhibitor, exhibited a potent cytotoxic effect on CCA cells by suppressing TOP2A expression in vitro (44). Thus, the therapeutic efficacy of Topo II inhibitors, such as andrographolide and anthracycline, in CCA patients should be further explored.

Polycomb repressive complex 1 (PRC1) is required for adult stem cell functions and acts as both a tumor suppressor and oncogene (45). Tang et al demonstrated the significant biological implications of PRC1 in tumor pathogenesis and prognosis in non-small cell lung cancer patients by analyzing genome-wide RNAi data and mRNA expression data (46). Bmi1 and EZH2 are representative members of PRC1. Sasaki et al found that Bmil was overexpressed in CCA cell lines and stimulated cell proliferation (47). Overexpression of EZH2 may induce hypermethylation of the $\mathrm{p} 16^{\mathrm{INK} 4 \mathrm{a}}$ promoter, followed by decreased expression of $16^{\mathrm{INK} 4 \mathrm{a}}$ in multistep cholangiocarcinogenesis (48). However, the precise molecular mechanisms underlying the role of $\mathrm{PRC} 1$ in CCA remain unclear.

Importantly, we noted that both CDK1 and PRC1 were involved in the same GO term, midbody. Midbody is a transient structure during cytokinesis and is involved in recruitment and organization of abscission machinery, which physically regulates the localization of two daughter cells (49). Midbody dysregulation causes mitotic problems in daughter cell separation, which increases cancer susceptibility and tumorigenesis (50). CITRON, a known serine kinase present at midbody during cytokinesis, could contribute to tumor occurrence in HCC (51). CDK1 phosphorylates septin 9 (SEPT9), thus playing an important role in mediating the final separation of daughter cells (52). Moreover, PCR1 could accumulate in the midbody during cytokinesis and organize the midbody through microtubule regulation (49). Based on this study, we may speculate that CDK1 and PRC1 contribute to the progression of CCA through midbody-related function.

Although we cannot exclude the possibility that the identified hub genes may be implicated in noncarcinogenic aspects of CCA, we attempted to ensure the credibility of the results by including as many datasets as possible. Except for TCGA, a total of 4 datasets were used to validate the differential expression of hub genes. In addition, the survival analysis of certain hub genes was based on 2 datasets. Moreover, we performed RT-qPCR to verify the selected hub genes based on 10 pairs of tissue samples.

In conclusion, we identified a number of hub genes and comprehensively revealed the biological functions and signaling pathways associated with CCA carcinogenesis through systematic bioinformatic analyses. Moreover, we identified $C D K 1, M K I 67, T O P 2 A$ and $P R C 1$ as possible prognostic biomarkers and further discussed the roles that the four genes may play in cancer development. Most of the genes have not been thoroughly studied in CCA. In future research, the clinical application of the identified hub genes as biomarkers for supervising the prognosis of CCA patients should be further investigated. Moreover, research concerning specific mechanisms of these genes in CCA occurrence and progression is warranted.

\section{Acknowledgements}

Not applicable.

\section{Funding}

This research was funded by the Beijing Municipal Science \& Technology Commission ResearchFund (no.Z171100000417004) and Beijing Natural Science Foundation (no. L172055).

\section{Availability of data and materials}

Data for CCA mRNA expression were downloaded from TCGA database (https://portal.gdc.cancer.gov/, RNA-seq, Illumina), Gene Expression Omnibus (GEO) database of the National Center for Biotechnology Information (GSE26566, GSE76297), and ArrayExpress Archive of Functional Genomics Data (E-GEOD-32879 and E-GEOD-45001). Clinical data of 118 patients with cholangiocarcinoma were downloaded from PubMed Central (PMID: 28667006). Matching sample information was obtained from GEO dataset: GSE89749. CCA tissue samples were collected from 10 patients with iCCA undergoing surgery at Peking Union Medical College Hospital. All patients were enrolled from November 2018 to April 2019.

\section{Authors' contributions}

HL, JuL and YZ designed this study. HL, JuL, FX, KK, YS, WX, XW, JiL, HX, SD, YX, HZ, YZ, and JG contributed to data curation and analysis. HL and WX conducted the laboratory experiment. HL, YS and XW wrote the manuscript. JuL and YZ revised the manuscript. All the authors read and approved the final version of the manuscript for publication and agree to be accountable for all aspects of the research in ensuring that the accuracy or integrity of any part of the work are appropriately investigated and resolved.

\section{Ethics approval and consent to participate}

The study was approved by the Clinical Research Ethics Committee of Peking Union Medical College Hospital. Each patient provided written signed informed consent.

\section{Patient consent for publication}

Not applicable.

\section{Competing interests}

The authors declare that they have no competing interests.

\section{References}

1. Razumilava N and Gores GJ: Cholangiocarcinoma. Lancet 383: 2168-2179, 2014.

2. Zhang H, Shen F, Han J, Shen YN, Xie GQ, Wu MC and Yang T: Epidemiology and surgical management of intrahepatic cholangiocarcinoma. Hepat Oncol 3: 83-91, 2016.

3. Bergquist A and von Seth E: Epidemiology of cholangiocarcinoma. Best Pract Res Clin Gastroenterol 29: 221-232, 2015.

4. Hu J and Yin B: Advances in biomarkers of biliary tract cancers. Biomed Pharmacother 81: 128-135, 2016. 
5. Esnaola NF, Meyer JE, Karachristos A, Maranki JL, Camp ER and Denlinger CS: Evaluation and management of intrahepatic and extrahepatic cholangiocarcinoma. Cancer 122: 1349-1369, 2016.

6. Squadroni M, Tondulli L, Gatta G, Mosconi S, Beretta G and Labianca R: Cholangiocarcinoma. Crit Rev Oncol Hematol 116: 11-31, 2017

7. Zhu AX: Future directions in the treatment of cholangiocarcinoma. Best Pract Res Clin Gastroenterol 29: 355-361, 2015.

8. Rizvi S and Gores GJ: Emerging molecular therapeutic targets for cholangiocarcinoma. J Hepatol 67: 632-644, 2017.

9. Mertens JC, Rizvi S and Gores GJ: Targeting cholangiocarcinoma. Biochim Biophys Acta Mol Basis Dis 1864: 1454-1460, 2018.

10. Ghouri YA, Mian I and Blechacz B: Cancer review: Cholangiocarcinoma. J Carcinog 14: 1, 2015

11. Jusakul A, Cutcutache I, Yong CH, Lim JQ, Huang MN, Padmanabhan N, Nellore V, Kongpetch S, Ng AWT, Ng LM, Choo SP, et al: Whole-genome and epigenomic landscapes of etiologically distinct subtypes of cholangiocarcinoma. Cancer Discov 7: 1116-1135, 2017.

12. Tomczak K, Czerwińska $P$ and Wiznerowicz M: The cancer genome atlas (TCGA): An immeasurable source of knowledge. Contemp Oncol (Pozn) 19: A68-A77, 2015.

13. Szklarczyk D, Franceschini A, Wyder S, Forslund K, Heller D, Huerta-Cepas J, Simonovic M, Roth A, Santos A, Tsafou KP, et al: STRING v10: Protein-protein interaction networks, integrated over the tree of life. Nucleic Acids Res 43 (Database Issue): D447-D452, 2015 .

14. Zheng Y, Long J, Wu L, Zhang H, Li L, Zheng Y, Wang A, Lin J, Yang X, Sang X, et al: Identification of hub genes involved in the development of hepatocellular carcinoma by transcriptome sequencing. Oncotarget 8: 60358-60367, 2017.

15. Wang X, Hu KB,Zhang YQ, Yang CJ and Yao HH: Comprehensive analysis of aberrantly expressed profiles of lncRNAs, miRNAs and mRNAs with associated ceRNA network in cholangiocarcinoma. Cancer Biomarkers 23: 549-559, 2018

16. Anders S and Huber W: Differential expression analysis for sequence count data. Genome Biol 11: R106, 2010.

17. Team RDC: R: A language and environment for statistical computing. Journal, 2010

18. Walter W, Sánchez-Cabo F and Ricote M: GOplot: An R package for visually combining expression data with functional analysis. Bioinformatics 31: 2912-2914, 2015.

19. Choi JK, Yu U, Yoo OJ and Kim S: Differential coexpression analysis using microarray data and its application to human cancer. Bioinformatics 21: 4348-4355, 2005.

20. Chaisaingmongkol J, Budhu A, Dang H, Rabibhadana S Pupacdi B, Kwon SM, Forgues M, Pomyen Y, Bhudhisawasdi V, Lertprasertsuke N, et al: Common molecular subtypes among asian hepatocellular carcinoma and cholangiocarcinoma. Cancer Cell 32: 57-70.e53, 2017.

21. Andersen JB, Spee B, Blechacz BR, Avital I, Komuta M, Barbour A, Conner EA, Gillen MC, Roskams T, Roberts LR, et al: Genomic and genetic characterization of cholangiocarcinoma identifies therapeutic targets for tyrosine kinase inhibitors. Gastroenterology 142: 1021-1031.e15, 2012.

22. Oishi N, Kumar MR, Roessler S, Ji J, Forgues M, Budhu A, Zhao X, Andersen JB, Ye QH, Jia HL, et al: Transcriptomic profiling reveals hepatic stem-like gene signatures and interplay of miR-200c and epithelial-mesenchymal transition in intrahepatic cholangiocarcinoma. Hepatology 56: 1792-1803, 2012

23. Sulpice L, Rayar M, Desille M, Turlin B, Fautrel A, Boucher E, Llamas-Gutierrez F, Meunier B, Boudjema K, Clément B and Coulouarn C: Molecular profiling of stroma identifies osteopontin as an independent predictor of poor prognosis in intrahepatic cholangiocarcinoma. Hepatology 58: 1992-2000, 2013.

24. Sulpice L, Desille M, Turlin B, Fautrel A, Boudjema K, Clément B and Coulouarn C: Gene expression profiling of the tumor microenvironment in human intrahepatic cholangiocarcinoma. Genom Data 7: 229-232, 2016.

25. Ritchie ME, Phipson B, Wu D, Hu Y, Law CW, Shi W and Smyth GK: Limma powers differential expression analyses for RNA-sequencing and microarray studies. Nucleic Acids Res 43: e47, 2015.

26. Robin X, Turck N, Hainard A, Tiberti N, Lisacek F, Sanchez JC and Müller M: pROC: An open-source package for R and S+ to analyze and compare ROC curves. BMC Bioinformatics 12: 77, 2011 .
27. Therneau TM: A package for survival analysis in S. version 2.38 , 2015, https://CRAN.R-project.org/package=survival.

28. Terry M and Therneau PMG: Modeling Survival data: Extending the Cox Model. Dietz K, Gail M, Krickeberg K, Samet J and Tsiatis A (eds). Springer, New York, NY, 2000.

29. Holt LJ, Tuch BB, Villen J, Johnson AD, Gygi SP and Morgan DO: Global analysis of Cdk1 substrate phosphorylation sites provides insights into evolution. Science 325: 1682-1686, 2009.

30. Ravindran Menon D, Luo Y, Arcaroli JJ,Liu S, KrishnanKutty LN, Osborne DG, Li Y, Samson JM, Bagby S, Tan AC, et al: CDK1 Interacts with Sox2 and promotes tumor initiation in human melanoma. Cancer Res 78: 6561-6574, 2018.

31. Okumura E, Fukuhara T, Yoshida H, Hanada Si S, Kozutsumi R, Mori M, Tachibana K and Kishimoto T: Akt inhibits Myt1 in the signalling pathway that leads to meiotic G2/M-phase transition. Nat Cell Biol 4: 111-116, 2002.

32. Wattanawongdon W, Hahnvajanawong C, Namwat $\mathrm{N}$, Kanchanawat S, Boonmars T, Jearanaikoon P, Leelayuwat C, Techasen A and Seubwai W: Establishment and characterization of gemcitabine-resistant human cholangiocarcinoma cell lines with multidrug resistance and enhanced invasiveness. Int J Oncol 47: 398-410, 2015.

33. Booth DG, Takagi M, Sanchez-Pulido L, Petfalski E, Vargiu G, Samejima K, Imamoto N, Ponting CP, Tollervey D, Earnshaw WC and Vagnarelli P: Ki-67 is a PP1-interacting protein that organises the mitotic chromosome periphery. Elife 3: e01641, 2014.

34. Klöppel G and La Rosa S: Ki67 labeling index: Assessment and prognostic role in gastroenteropancreatic neuroendocrine neoplasms. Virchows Arch 472: 341-349, 2018

35. Miller HC, Drymousis P, Flora R, Goldin R, Spalding D and Frilling A: Role of Ki-67 proliferation index in the assessment of patients with neuroendocrine neoplasias regarding the stage of disease. World J Surg 38: 1353-1361, 2014.

36. Singh S, Hallet J, Rowsell C and Law CHL: Variability of Ki67 labeling index in multiple neuroendocrine tumors specimens over the course of the disease. Eur J Surg Oncol 40: 1517-1522, 2014.

37. Grillo F, Albertelli M, Brisigotti MP, Borra T, Boschetti M, Fiocca R, Ferone D and Mastracci L: Grade increases in gastroenteropancreatic neuroendocrine tumor metastases compared to the primary tumor. Neuroendocrinology 103: 452-459, 2016.

38. Romero A, Martín M, Cheang MC, López García-Asenjo JA, OlivaB,HeX, delaHoya M,GarcíaSáenzJÁ, ArroyoFernándezM, Díaz Rubio E, et al: Assessment of Topoisomerase II $\alpha$ status in breast cancer by quantitative PCR, gene expression microarrays, immunohistochemistry, and fluorescence in situ hybridization. Am J Pathol 178: 1453-1460, 2011.

39. Järvinen TA and Liu LE: Simultaneous amplification of HER-2 (ERBB2) and topoisomerase IIalpha (TOP2A) genes-molecular basis for combination chemotherapy in cancer. Curr Cancer Drug Targets 6: 579-602, 2006.

40. Liang Z, Zeng X, Gao J, Wu S, Wang P, Shi X, Zhang J and Liu T: Analysis of EGFR, HER2, and TOP2A gene status and chromosomal polysomy in gastric adenocarcinoma from Chinese patients. BMC Cancer 8: 363, 2008.

41. Press MF, Sauter G, Buyse M, Bernstein L, Guzman R, Santiago A, Villalobos IE, Eiermann W, Pienkowski T, Martin M, et al: Alteration of topoisomerase II-alpha gene in human breast cancer: Association with responsiveness to anthracycline-based chemotherapy. J Clin Oncol 29: 859-867, 2011.

42. Simon R, Atefy R, Wagner U, Forster T, Fijan A, Bruderer J, Wilber K, Mihatsch MJ, Gasser T and Sauter G: HER-2 and TOP2A coamplification in urinary bladder cancer. Int J Cancer 107: 764-772, 2003.

43. Panvichian R, Tantiwetrueangdet A, Angkathunyakul N and Leelaudomlipi S: TOP2A amplification and overexpression in hepatocellular carcinoma tissues. Biomed Res Int 2015: 381602, 2015.

44. Nateewattana J, Dutta S, Reabroi S, Saeeng R, Kasemsook S, Chairoungdua A, Weerachayaphorn J, Wongkham $S$ and Piyachaturawat P: Induction of apoptosis in cholangiocarcinoma by an andrographolide analogue is mediated through topoisomerase II alpha inhibition. Eur J Pharmacol 723: 148-155, 2014.

45. Gil J and O'Loghlen A: PRC1 complex diversity: Where is it taking us? Trends Cell Biol 24: 632-641, 2014.

46. Tang H, Xiao G, Behrens C, Schiller J, Allen J, Chow CW, Suraokar M, Corvalan A, Mao J, White MA, et al: A 12-gene set predicts survival benefits from adjuvant chemotherapy in non-small cell lung cancer patients. Clin Cancer Res 19: $1577-1586,2013$ 
47. Sasaki M, Yamaguchi J, Ikeda H, Itatsu K and Nakanuma Y: Polycomb group protein Bmil is overexpressed and essential in anchorage-independent colony formation, cell proliferation and repression of cellular senescence in cholangiocarcinoma: Tissue and culture studies. Hum Pathol 40: 1723-1730, 2009.

48. Sasaki M, Yamaguchi J, Itatsu K, Ikeda H and Nakanuma Y: Over-expression of polycomb group protein EZH2 relates to decreased expression of p16 INK4a in cholangiocarcinogenesis in hepatolithiasis. J Pathol 215: 175-183, 2008.

49. Hu CK, Coughlin M and Mitchison TJ: Midbody assembly and its regulation during cytokinesis. Mol Biol Cell 23: 1024-1034, 2012.

50. Sadler JBA, Wenzel DM, Williams LK, Guindo-Martínez M, Alam SL, Mercader JM, Torrents D, Ullman KS, Sundquist WI and Martin-Serrano J: A cancer-associated polymorphism in ESCRT-III disrupts the abscission checkpoint and promotes genome instability. Proc Natl Acad Sci USA 115: E8900-E8908, 2018.
51. Fu Y, Huang J, Wang KS, Zhang X and Han ZG: RNA interference targeting CITRON can significantly inhibit the proliferation of hepatocellular carcinoma cells. Mol Biol Rep 38: 693-702, 2011.

52. Estey MP, Di Ciano-Oliveira C, Froese CD, Fung KY, Steels JD, Litchfield DW and Trimble WS: Mitotic regulation of SEPT9 protein by cyclin-dependent kinase $1(\mathrm{Cdk} 1)$ and Pin 1 protein is important for the completion of cytokinesis. J Biol Chem 288: 30075-30086, 2013.

This work is licensed under a Creative Commons Attribution-NonCommercial-NoDerivatives 4.0 International (CC BY-NC-ND 4.0) License. 